PROCEEDINGS OF THE

AMERICAN MATHEMATICAL SOCIETY

Volume 136, Number 1, January 2008, Pages 311-320

S 0002-9939(07)08716-3

Article electronically published on October 12, 2007

\title{
ON SCH AND THE APPROACHABILITY PROPERTY
}

\author{
MOTI GITIK AND ASSAF SHARON
}

(Communicated by Julia Knight)

\begin{abstract}
We construct a model of $\neg S C H+\urcorner A P+$ (Very Good Scale). This answers questions of Cummings, Foreman, Magidor and Woodin.
\end{abstract}

\section{INTRODUCTION}

Notions of Very Good Scale $_{\kappa}\left(V G S_{\kappa}\right)$, Weak square ${ }_{\kappa}\left(\square_{\kappa}^{*}\right)$ and the Approachability Property ${ }_{\kappa}\left(A P_{\kappa}\right)$, for a singular $\kappa$, play a central role in Singular Cardinals Combinatorics. They were extensively studied by Shelah [9, 10, 11] and by Cummings, Foreman and Magidor 2].

All of these properties break down above a supercompact cardinal as was shown by S. Shelah in 9]. By R. Solovay [12, the Singular Cardinal Hypothesis (SCH) holds above strong compact cardinals. Also by Ben-David and Magidor [1 the Prikry forcing adds $\square_{\kappa}^{*}$. Hence it is natural to ask about interconnections between $\mathrm{SCH}$ and the above principles. Cummings, Foreman and Magidor [2] asked if $V G S_{\kappa}$ implies $\square_{\kappa}^{*}$. Woodin previously asked if it is possible to have $\neg S C H_{\kappa}+\neg \square_{\kappa}^{*}$. In 4 ] the positive answer to the second question was claimed. The second author found a gap in the argument and was able to show that the forcing used there (extender based forcing with long extenders) actually adds a $\square_{\kappa}^{*}$ - sequence.

Our goal here will be to give a negative answer to the first question and a positive answer to the second. Thus we prove the following:

Theorem 1.1. Suppose $\kappa$ is a supercompact cardinal. Then there is a generic extension in which $\kappa$ is a strong limit singular cardinal of cofinality $\omega$ so that

(a) $2^{\kappa}>\kappa^{+}$;

(b) $\neg A P_{\kappa}$ (and hence $\urcorner \square_{\kappa}^{*}$ );

(c) $V G S_{\kappa}$.

Using standard methods we can make $\kappa$ into $\aleph_{\omega^{2}}$. Namely the following holds:

Theorem 1.2. Suppose $\kappa$ is a supercompact cardinal. Then there is a generic extension in which $\kappa=\aleph_{\omega^{2}}$ is a strong limit cardinal so that

Received by the editors April 17, 2005 and, in revised form, December 5, 2005 and March 14, 2006.

2000 Mathematics Subject Classification. Primary 03E35, 03E55.

The authors are grateful to John Krueger, James Cummings and the referee for their remarks and corrections. 
(a) $2^{\aleph_{\omega^{2}}}>\aleph_{\omega^{2}+1}$

(b) $\neg A P_{\aleph_{\omega^{2}}}$;

(c) $V G S_{\aleph_{\omega^{2}}}$.

\section{THE MAIN CONSTRUCTION}

Let us first recall some basic definitions:

Definition 2.1. (S. Shelah [9]) A sequence $\left\langle C_{\alpha} \mid \alpha<\kappa^{+}\right\rangle$is called an $A P_{\kappa}$-sequence iff

(a) $\lim (\alpha) \rightarrow C_{\alpha}$ is a club in $\alpha$ and o.t. $\left(C_{\alpha}\right)=c f(\alpha)$.

(b) There is a club subset $D$ of $\kappa^{+}$such that

$$
\forall \alpha \in D \forall \beta<\alpha \exists \gamma<\alpha C_{\alpha} \cap \beta=C_{\gamma} .
$$

It is not hard to see that $\square_{\kappa}^{*} \rightarrow A P_{\kappa}$.

Definition 2.2. (a) Let $\left\langle\kappa_{n} \mid n<\omega\right\rangle$ be a sequence of regular cardinals such that $\bigcup_{n<\omega} \kappa_{n}=\kappa$. A sequence $\left\langle f_{\alpha} \mid \alpha<\kappa^{+}\right\rangle \subseteq \prod_{n<\omega} \kappa_{n}$ is called a very good scale on $\prod_{n<\omega} \kappa_{n}$ iff

(i) $\left\langle f_{\alpha} \mid \alpha<\kappa^{+}\right\rangle$is a scale on $\prod_{n<\omega} \kappa_{n}$, i.e., for every $f \in \prod_{n<\omega} \kappa_{n}$ there exists $\beta<\kappa^{+}$and $n<\omega$ such that $f(m)<f_{\beta}(m)$ for every $m>n$ and for every $\alpha<\beta<\kappa^{+}, f_{\alpha}(m)<f_{\beta}(m)$ for almost every $m$;

(ii) for every $\beta<\kappa^{+}$such that $\omega<\operatorname{cf}(\beta)$ there exists a club $C$ of $\beta$ and $n<\omega$ such that $f_{\gamma_{1}}(m)<f_{\gamma_{2}}(m)$ for every $\gamma_{1}<\gamma_{2} \in C$ and $m>n$.

(b) $V G S_{\kappa}$ holds iff there exists a sequence $\left\langle\kappa_{n} \mid n<\omega\right\rangle$ and $\left\langle f_{\alpha} \mid \alpha<\kappa^{+}\right\rangle$such that $\left\langle f_{\alpha} \mid \alpha<\kappa^{+}\right\rangle$is a very good scale on $\prod_{n<\omega} \kappa_{n}$.

Definition 2.3. (S. Shelah 9]) Let $\kappa$ be an uncountable cardinal such that $c f(\kappa)=$ $\omega$, and $d:\left[\kappa^{+}\right]^{2} \rightarrow \omega$.

(a) $d$ is called normal if $\forall \beta \forall n<\omega|\{\alpha<\beta \mid d(\alpha, \beta) \leq n\}|<\kappa$.

(b) $d$ is called subadditive if $\forall \alpha<\beta<\gamma<\kappa^{+}, d(\alpha, \gamma) \leq \max (d(\alpha, \beta), d(\beta, \gamma))$.

(c) $S_{0}(d)=\left\{\alpha<\kappa^{+} \mid \exists A, B \subseteq \alpha\right.$ unbounded in $\alpha$ such that

$$
\forall \beta \in B \exists n_{\beta} \in \omega \forall \alpha \in A \cap \beta d(\alpha, \beta) \leq n_{\beta} .
$$

The next Lemma, which was stated in Shelah 9, shows that such a function always exists. Let us give the proof for the benefit of the reader.

Lemma 2.4. (S.Shelah 9]) There exists a normal subadditive function $d:\left[\kappa^{+}\right]^{2} \rightarrow$ $\omega$ for every uncountable cardinal $\kappa$ such that $c f(\kappa)=\omega$.

Proof. Fix an increasing sequence $\left\langle\kappa_{n} \mid n<\omega\right\rangle$ of regular cardinals cofinal in $\kappa$. For every $d:\left[\kappa^{+}\right]^{2} \rightarrow \omega$, let $A(\beta, n)$ and $(A(\beta, \leq n))$ denote the set of all $\gamma<\beta$ such that $d(\gamma, \beta)=n$ and $d(\gamma, \beta) \leq n$ respectively. We are going to define the function $d \Gamma_{\gamma \times \gamma}$ by induction on $\gamma$ such that for every $\beta \geq \kappa$ the size of $A(\beta, n)$ is at most $\kappa_{n}$. For every $\gamma<\beta<\kappa$, we define $d(\gamma, \beta)$ to be the least $n$ such that $\gamma<\kappa_{n}$. Assume that $d \Gamma_{\gamma \times \gamma}$ is defined. If $\gamma=\eta+1$ is a successor, then let $d(\alpha, \gamma)=d(\alpha, \eta)$ for every $\alpha<\eta$ and $d(\eta, \gamma)=0$. It is simple to see that $d \Gamma_{\gamma \times \gamma}$ is normal and subadditive. Assume now that $\gamma$ is a limit ordinal. Let $\left\langle B_{i} \mid i<\omega\right\rangle$ be a $\subseteq$ - increasing sequence such that $\bigcup_{i<\omega} B_{i}=\gamma$ and $\left|B_{i}\right|=\kappa_{i}$. We define the sets $A(\gamma, n)$ by induction on $n$ as follows: By the induction hypothesis we can find $A(\gamma, 0)$ such that $B_{0} \subseteq A(\gamma, 0)$ 
and for every $\alpha \in A(\gamma, 0)$ the set $A(\alpha, 0)$ is contained in $A(\gamma, 0)$. Assume that $A(\gamma, n-1)$ is defined. Set

$$
X_{n}=\bigcup\left\{A(\alpha, n) \mid \alpha \in \bigcup_{i<n} A(\gamma, i)\right\} .
$$

Note that by the induction hypothesis $\left|X_{n}\right| \leq \kappa_{n}$. By another application of the induction hypothesis, it is possible to find $Y_{n} \supseteq X_{n} \cup B_{n}$ of size $\kappa_{n}$ such that $A(\alpha, \leq n) \subseteq Y_{n}$ for every $\alpha \in Y_{n}$. Let $A(\gamma, n)=Y_{n}-\bigcup_{i<n} A(\gamma, i)$. Note that the size of $A(\gamma, n)$ is $\kappa_{n}$. Now define

$$
d(\alpha, \gamma)=n \text { iff } \alpha \in A(\gamma, n) .
$$

Let us show that the function $d \uparrow_{\gamma \times \gamma}$ is subadditive: Let $\beta<\alpha<\gamma$. Set $n=d(\alpha, \gamma)$ and $k=d(\beta, \alpha)$. We consider two cases:

Case 1: $n \geq k$. But then by our construction, $\beta \in \bigcup_{i \leq n} A(\gamma, i)$ and so $d(\beta, \gamma) \leq$ $n$.

Case 2: $n<k$. But then $\beta \in X_{k}$ and so $\beta \in Y_{k}$ and $d(\beta, \gamma) \leq k$.

This finishes the proof of the lemma.

Fact 2.5. (S. Shelah 9 ) Suppose that $\kappa$ is a strong limit cardinal of cofinality $\omega$ and $d, d^{\prime}:\left[\kappa^{+}\right]^{2} \rightarrow \omega$ are two normal functions. Then $S_{0}(d) \equiv S_{0}\left(d^{\prime}\right)\left(\bmod \mathcal{D}_{\kappa^{+}}\right)$ (where $\mathcal{D}_{\kappa^{+}}$is the club filter).

Fact 2.6. (S. Shelah 9]) Let $\kappa$ be a singular strong limit cardinal of cofinality $\omega$. The statement $A P_{\kappa}$ is equivalent to the existence of a normal function $d:\left[\kappa^{+}\right]^{2} \rightarrow \omega$ such that $S_{0}(d)$ contains a club.

$S_{0}(d)$ is in fact the set of all approachable points and $A P_{\kappa}$ means that modulo the club filter every point less than $\kappa^{+}$is approachable.

Let us now prove Theorem 1.1. We start with a model $V$ of $Z F C+G C H$ such that $V \models$ " $\kappa$ is supercompact". Iterate first in Backward Easton fashion the Cohen forcing $C\left(\alpha, \alpha^{+\omega+2}\right)$ for each inaccessible $\alpha \leq \kappa$, where $C\left(\alpha, \alpha^{+\omega+2}\right)$ is defined as the poset consisting of functions $f$ such that $\operatorname{Dom}(f)$ is a subset of $\alpha^{+\omega+2}$ of size less than $\alpha$ and for every $\beta \in \operatorname{Dom}(f), f(\beta)$ is a partial function from $\alpha$ to $\alpha$ of size less than $\alpha$.

Let $\mathcal{P}_{<\kappa}$ denote the iteration below $\kappa$ and $\mathcal{P}_{\kappa}=\mathcal{P}_{<\kappa} * C\left(\kappa, \kappa^{+\omega+2}\right)$. Note that the forcing $\mathcal{P}_{\kappa}$ preserves the cofinality of the ordinals. Let $G$ be a generic subset of $\mathcal{P}_{\kappa}$. Denote $G_{<\kappa}=\mathcal{P}_{<\kappa} \cap G$. Let for each $\alpha<\kappa^{+\omega+2}, F_{\alpha}$ denote the $\alpha$-th generic function from $\kappa$ to 2 in $G$, i.e. $\bigcup\{f(\alpha) \mid f \in G\}$.

Fix in $V$ a normal ultrafilter $U$ over $P_{\kappa}\left(\kappa^{+\omega+2}\right)$. Let $j: V \rightarrow M \simeq U l t(V, U)$ be the corresponding elementary embedding. Then $\operatorname{crit}(j)=\kappa$ and ${ }^{\kappa^{+\omega+2}} M \subseteq M$.

By standard arguments (see [6]) $j$ extends in $V[G]$ to an elementary embedding $j^{*}: V[G] \rightarrow M\left[G^{*}\right]$, where $G^{*} \cap \mathcal{P}_{\kappa}=G_{\kappa}$ and $G^{*}$ above $\kappa$ is constructed in $V[G]$ using closure of the forcing and the fact that the number of dense sets we need to meet is small. Also, over $j(\kappa)$, we need to start with the condition $\left\{\left\langle j(\alpha), F_{\alpha}\right\rangle \mid\right.$ $\left.\alpha<\kappa^{+\omega+2}\right\}$ in order to satisfy $j^{\prime \prime} G \subseteq G^{*}$. This means that for each $\alpha<\kappa^{+\omega+2}$ the function $F_{j(\alpha)}$ (i.e. the one $G^{*}$ defines to be $j(\alpha)$-th function from $j(\kappa)$ to $j(\kappa)$ ) should extend $F_{\alpha}$.

Note that above $\kappa$ we are free in choosing values of $F_{j(\alpha)}$. Let us require $F_{j(\alpha)}(\kappa)=\alpha$ for each $\alpha<\kappa^{+\omega+2}$ and then continue to build $G^{*}$.

Let $U^{*}=\left\{X \subseteq P_{\kappa}\left(\kappa^{+\omega+2}\right) \mid j^{\prime \prime} \kappa^{+\omega+2} \in j^{*}(X)\right\}$. Then $U^{*} \supseteq U$ and it is a normal ultrafilter over $P_{\kappa}\left(\kappa^{+\omega+2}\right)$ in $V[G]$. 
Lemma 2.7. (1) For every $\xi<\rho<\kappa^{+\omega+2}\left\{P \in P_{\kappa}\left(\kappa^{+\omega+2}\right) \mid F_{\xi}(P \cap \kappa)<\right.$ $\left.F_{\rho}(P \cap \kappa)\right\} \in U^{*}$.

(2) For each $f \in \prod\left\{\delta^{+\omega+1} \mid \delta<\kappa, \delta\right.$ is an inaccessible $\}$ there is $\xi<\kappa^{+\omega+1}$ such that

$$
\left\{P \in P_{\kappa}\left(\kappa^{+\omega+1}\right) \mid f(P \cap \kappa)=F_{\xi}(P \cap \kappa)\right\} \in U^{*} .
$$

Proof. (1) In $M\left[G^{*}\right]$, we have $j^{*}\left(F_{\xi}\right)(\kappa)=\xi<j^{*}\left(F_{\rho}\right)(\kappa)=\rho$. Hence the conclusion follows from the definition of $U^{*}$.

(2) Again, in $M\left[G^{*}\right]$, we have $j^{*}(f)(\kappa)<\kappa^{+\omega+1}$. Let $\xi=j^{*}(f)(\kappa)$. It is simple to see that $\xi$ satisfies the desired property.

For every $n \in \omega$ let $U_{n}$ be the projection of $U^{*}$ on $P_{\kappa}\left(\kappa^{+n}\right)$, i.e., $X \in U_{n}$ iff $\left\{P \in P_{\kappa}\left(\kappa^{+\omega+2}\right) \mid P \cap \kappa^{+n} \in X\right\} \in U^{*}$. Clearly $U_{n}$ is a normal ultrafilter on $\mathcal{P}_{\kappa}\left(\kappa^{+n}\right)$.

Let $a, b \in P_{\kappa}\left(\kappa^{+n}\right)$ and $b \cap \kappa \in \kappa$. Set

$$
a \subsetneq b \leftrightarrow(a \subseteq b) \wedge \text { o.t. }(p(a))<b \cap \kappa .
$$

Lemma 2.8. 7

(a) $\forall a \in P_{\kappa}\left(\kappa^{+n}\right)\left\{b \in P_{\kappa}\left(\kappa^{+n}\right) \mid a \subsetneq b\right\} \in U_{n}$.

(b) $\left\{a \in P_{\kappa}\left(\kappa^{+n}\right) \mid a \cap \kappa\right.$ is inaccessible and $\left.a \cap \kappa \in \kappa\right\} \in U_{n}$.

(c) Let $\vec{X}=\left\langle X_{a} \mid a \in P_{\kappa}\left(\kappa^{+n}\right)\right\rangle$ be a sequence of sets from $U_{n}$. Then $\Delta \vec{X}=$ $\left\{b \in P_{\kappa}\left(\kappa^{+n}\right) \mid \forall a \in P_{\kappa}\left(\kappa^{+n}\right) a \subsetneq b \rightarrow b \in X_{a}\right\} \in U_{n} .(\Delta \vec{X}$ is called the diagonal intersection of $\vec{X}$.)

We now define a version of the diagonal supercompact Prikry forcing.

Definition 2.9. $p \in Q$ iff $p=\left\langle a_{0}^{p}, a_{1}^{p}, \ldots, a_{n-1}^{p}, X_{n}^{p}, X_{n+1}^{p}, \ldots\right\rangle$ where

(i) $\forall \ell<n a_{\ell}^{p} \in P_{\kappa}\left(\kappa^{+\ell}\right)$ and $a_{\ell}^{p} \cap \kappa$ is an inaccessible cardinal;

(ii) $\forall m \geq n X_{m}^{p} \in U_{m}$;

(iii) $\forall m \geq n \forall b \in X_{m}^{p} \forall \ell<n a_{\ell}^{p} \subseteq b$;

(iv) $\forall i<j<n a_{i}^{p} \Subset a_{j}^{p}$.

$n$ is called the length of $p$ and will be denoted by $\ell(p)$.

Definition 2.10. Let $p, q \in Q$. Then $p \leq^{*} q$ iff

(i) $\ell(p)=\ell(q)$;

(ii) $\forall \ell<\ell(p) a_{\ell}^{p}=a_{\ell}^{q}$;

(iii) $\forall m \geq \ell(p) X_{m}^{q} \subseteq X_{m}^{p}$.

Definition 2.11. Suppose that $p \in Q$ and $\vec{a}=\langle\vec{a}(\ell(p)), \cdots, \vec{a}(m)\rangle$ where $\vec{a}(i) \in X_{i}^{p}$ for every $\ell(p) \leq i \leq m$. We denote by $p \frown\langle\vec{a}\rangle$ the sequence

$$
\left\langle a_{1}^{p}, \ldots, a_{\ell(p)-1}^{p}, \vec{a}(\ell(p)), \ldots, \vec{a}(m), Y_{m+1}, Y_{m+2}, \ldots\right\rangle,
$$

where

$$
Y_{n}=\left\{b \in X_{n}^{p} \mid \forall \ell(p) \leq i \leq m \vec{a}(i) \cong b\right\}
$$

for every $n \geq m+1$. 
By Lemma 2.8(a) it is easy to see that $Y_{k} \in U_{k}$, for each $k>m$ and $p \frown\langle\vec{a}\rangle \in Q$.

Definition 2.12. Let $p, q \in Q \cdot p \leq q$ iff there exists $\vec{a}$ such that $p \frown\langle\vec{a}\rangle \leq^{*} q$.

The proof of the next two claims is quite standard, and it uses the same arguments as in the case of the ordinary diagonal Prikry forcing notion; see [5].

Lemma 2.13. (a) $\left\langle Q \leq, \leq^{*}\right\rangle$ is a Prikry type forcing notion, i.e., if $\sigma$ is a statement in the forcing language, then for every $p \in P$ there exists $p \leq^{*} q \in P$ such that $q$ forces $\sigma$ or $\neg \sigma$.

(b) $\left\langle Q, \leq^{*}\right\rangle$ is $\kappa$-closed.

Proof. (a) Assume for simplicity that $\ell(p)=0$. Let $\sigma$ be a statement in the forcing language. Since any two conditions of length 0 are compatible, it is sufficient to find a condition $q$ such that $\ell(q)=0$ and $q$ decides $\sigma$. Let $\vec{a}=\left\langle a_{0}, \ldots, a_{n}\right\rangle$ be such that $a_{i} \in P_{\kappa}\left(\kappa^{i}\right)$ for every $i \leq n$ and $a_{i} \subsetneq a_{i+1}$ for every $i<n$. Define a sequence $X_{\vec{a}}$ as follows: If there exists a sequence $\vec{X}=\left\langle X_{m} \mid m \geq n+1\right\rangle$ such that $\vec{a} \frown \vec{X}$ is in $Q$ and decides $\sigma$, then let $X_{\vec{a}}$ be such a sequence. Otherwise let $X_{\vec{a}}(m)=P_{\kappa}\left(\kappa^{+m}\right)$ for every $m \geq n+1$. Using Lemma 2.8 (c), we can find $Y_{n} \in U_{n}$ such that for every $\subsetneq$ increasing sequence $\vec{a}=\left\langle a_{0}, \ldots, a_{n}\right\rangle$ and for every $m \geq n+1$,

$$
\left\{b \in Y_{m} \mid a_{n} \subsetneq b\right\} \subseteq X_{\vec{a}}(m) .
$$

Using Lemma 2.8 again, we can find a condition $q=\left\langle Y_{0}^{\prime}, Y_{1}^{\prime}, \ldots,\right\rangle$ such that $Y_{i}^{\prime} \subseteq Y_{i}$ with the following property: if there exists $\vec{a} \in \prod_{i \leq n} Y_{i}^{\prime}$ such that $q \frown\langle\vec{a}\rangle$ decides $\sigma$, then $q \frown\langle\vec{a}\rangle$ decides $\sigma$ for every $\vec{a} \in \prod_{i \leq n} Y_{i}^{\prime}$ (in the same way). Now it is easy to see that $q$ decides $\sigma$ and is of length 0 .

(b) This is an immediate consequence of the $\kappa$ completeness of the ultrafilters.

Lemma 2.14. Let $G_{Q}$ be $Q$ generic over $V[G]$.

(a) $\langle Q, \leq\rangle$ does not add any new bounded subsets to $\kappa$.

(b) $\forall n c f^{V[G]\left[G_{Q}\right]}\left(\kappa^{+n}\right)=\omega$ (in fact for every $\kappa \leq \delta<\kappa^{+\omega}$ such that $c f^{V[G]}(\delta)$ $\geq \kappa$ we have $\left.c f^{V[G]\left[G_{Q}\right]}(\delta)=\omega\right)$.

Proof. (a) This is a consequence of Lemma 2.13.

(b) Let $\left\langle a_{0}, a_{1}, \ldots\right\rangle$ be the generic sequence added by $G_{Q}$. Let $\delta<\kappa^{+\omega}$ be such that $c f^{V[G]}(\delta) \geq k$. A simple density argument shows that the sequence $\gamma_{m}=\sup \left(a_{m} \cap \delta\right)$ is cofinal in $\delta$.

The next lemma is crucial for the construction.

Lemma 2.15. $Q_{3}$ is $\kappa^{+\omega+1}-$ c.c.

Proof. Just note that the total number of finite sequences used in the conditions is $\kappa^{+\omega}$.

The next lemma now follows easily.

Lemma 2.16. (a) $V[G]\left[G_{Q}\right] \models$ " $\kappa$ is strong limit, $2^{\kappa}=\kappa^{+2}=\left(\kappa^{+\omega+2}\right)^{V[G]}$ and $c f(\kappa)=\omega "$.

(b) If $V[G]\left[G_{Q}\right] \models$ " $\omega<\mu=c f(\mu)<\kappa$ and $f: \mu \rightarrow V[G]$ ", then there is $X \in V[G]$ unbounded in $\mu$ such that $f \uparrow X \in V[G]$. 
Proof. (b): Let $\dot{f}$ be a $Q$ name for $f$. Let $D$ be the set of all conditions $p$ in $P$

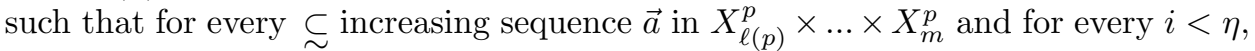
if there exists $p^{-}\langle\vec{a}\rangle \leq^{*} q \in P$ such that $q$ decides the value of $\dot{f}(i)$, then $p^{\frown}\langle\vec{a}\rangle$ already decides the value of $\dot{f}(i)$. Let us show that $D$ is dense. Let $p$ be a condition in $P$. Assume for simplicity that $\ell(p)=0$. Using the fact that the ultrafilters $U_{n}$ are

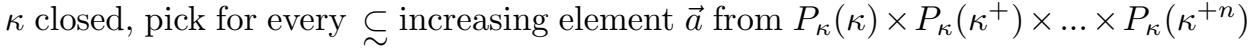
a condition $p_{\vec{a}}$ with initial segment $\vec{a}$ such that for every $i<\eta$ if there is a direct extension of $p_{\vec{a}}$ which decides the value of $\dot{f}(i)$, then $p_{\vec{a}}$ already decides this value. Using Lemma 2.8(c), find a condition $q$ such that $\ell(q)=0$ and $q \frown \vec{a} \geq^{*} p_{\vec{a}}$ for every $\subsetneq$ increasing sequence $\vec{a}$ in $X_{0}^{p} \times \ldots \times X_{m}^{p}$. Since every two conditions of length 0 are compatible, we can assume that $q \geq^{*} p$. But $q$ is in $D$ and so $D$ is dense in $P$.

Pick $p \in D \cap G_{Q}$ and let $p \Gamma_{\ell(p)} \vec{a}$ be the Prikry sequence added by $G_{Q}$. For every $i<\eta$ we can find $m(i)<\omega$ and $q \in G$ such that $q$ is a direct extension of $p \frown \vec{a} \uparrow_{m(i)}$ and $q$ decides the value of $\dot{f}(i)$. But then $p \frown \vec{a} \uparrow_{m(i)}$ already decides the value of $\dot{f}(i)$. Since $c f(\eta)>\omega$, we can find a stationary set $X^{\prime} \subseteq \eta$ and $m$ such that $m=m(i)$ for every $i$ in $X^{\prime}$. In $V[G]$ let

$$
X=\left\{i<\eta \mid p \frown \vec{a} \uparrow_{m} \text { decides the value of } \dot{f}(i)\right\} .
$$

Then $X$ is as required.

Definition 2.17. A submodel $N$ of $H_{\kappa^{+\omega+1}}$ is called a supercompact submodel iff

(1) $|N|<\kappa$ and $N \cap \kappa$ is a cardinal less than $\kappa$;

(2) $c f\left(\sup \left(N \cap \kappa^{+\omega+1}\right)=(N \cap \kappa)^{+\omega+1}\right.$;

(3) for every $A \subseteq \kappa^{+\omega+1}$ there exists $B \in N$ such that

$$
A \cap N=B \cap N .
$$

It is simple to see that if $\kappa$ is $\kappa^{+\omega+2}$ supercompact, then the collection of all supercompact submodels is stationary. The following lemma was proved by Shelah in $[9]$ :

Lemma $2.18([9])$. Suppose that $\kappa$ is $\kappa^{+\omega+2}$ supercompact and $d:\left[\kappa^{+\omega+1}\right]^{2} \rightarrow \omega$ is normal and subadditive. Let $S$ be the set of $\delta<\kappa^{+\omega+1}$ such that $\delta=\sup \left(N \cap \kappa^{+\omega+1}\right)$ for some supercompact submodel. Then

$$
S \subseteq \kappa^{+\omega+1} \cap c f(<\kappa) \text { is stationary }
$$

and

$$
S \subseteq \kappa^{+\omega+1}-S_{0}(d)
$$

Let $G_{Q}$ be a generic subset of $Q$ over $V[G]$.

Proposition 2.19. $V[G]\left[G_{Q}\right] \models \neg A P_{\kappa}$.

Proof. The idea is to try to find a normal function $d$ such that $\kappa^{+}-S_{0}(d)$ is stationary. The next lemma shows that it is sufficient to find any two-place function $d$ with this property.

Lemma 2.20. Let $\kappa$ be a cardinal such that $c f(\kappa)=\omega$. If there is $d:\left[\kappa^{+}\right]^{2} \rightarrow \omega$ such that $\kappa^{+}-S_{0}(d)$ is stationary, then there is a normal $\bar{d}$ such that $\kappa^{+}-S_{0}(\bar{d})$ is stationary. 
Proof. Let $d_{0}:\left[\kappa^{+}\right]^{2} \rightarrow \omega$ be any normal function. Set $\bar{d}=d+d_{0}$. We need to show that $\bar{d}$ is normal and that $\kappa^{+}-S_{0}(\bar{d})$ is stationary.

(i) $\bar{d}$ is normal: pick $\beta<\kappa^{+}$. Since $\bar{d}(\alpha, \beta) \geq d_{0}(\alpha, \beta)$, we see that $\{\alpha<\beta \mid$ $\bar{d}(\alpha, \beta) \leq n\} \subseteq\left\{\alpha<\beta \mid d_{0}(\alpha, \beta) \leq n\right\}$ and the conclusion follows from the normality of $d_{0}$.

(ii) $\kappa^{+}-S_{0}(\bar{d})$ is stationary: for every $\beta \in S_{0}(\bar{d})$, there are $A, B \subseteq \beta$ unbounded in $\beta$ which satisfy Definition 2.3(c). Since $\forall \alpha<\beta d(\alpha, \beta) \leq \bar{d}(\alpha, \beta)$ we get $\beta \in S_{0}(d)$. We proved that $S_{0}(\bar{d}) \subseteq S_{0}(d)$ or equivalently $\kappa^{+}-S_{0}(\bar{d}) \supseteq$ $\kappa^{+}-S_{0}(d)$. But $\kappa^{+}-S_{0}(d)$ is stationary and so $\kappa^{+}-S_{0}(\bar{d})$ is also stationary.

Work in $V[G]$ and pick any normal subadditive function $d:\left[\kappa^{+\omega+1}\right]^{2} \rightarrow \omega$. Set $S=\kappa^{+}-\left(S_{0}(d)\right)^{V[G]}$. Since $\kappa$ is $\kappa^{+\omega+2}$ supercompact, we can apply Lemma 2.18 and conclude that $S$ is stationary. In $V\left[G, G_{Q}\right], d$ is a function from $\left[\kappa^{+}\right]^{2}$ to $\omega$, but $d$ is no longer normal. Let us prove that $V\left[G, G_{Q}\right] \models S \subseteq \kappa^{+}-S_{0}(d)$. Otherwise there exists $\delta \in S \cap S_{0}(d)$. Pick $A, B \in V\left[G, G_{Q}\right]$ unbounded in $\delta$ such that

$$
\forall \beta \in B \exists n_{\beta} \forall \alpha<\beta \alpha \in A \rightarrow d(\alpha, \beta) \leq n_{\beta} .
$$

Since $\omega<c f^{V\left[G, G_{Q}\right]}(\delta)<\kappa$, we can use Lemma 2.16(b) to find $\bar{A}, \bar{B} \in V[G]$ unbounded in $\delta$ such that $\bar{A} \subseteq A$ and $\bar{B} \subseteq B$. We have that for every $\beta$ in $\bar{B}$ there exists $n_{\beta}$ such that $d(\alpha, \beta) \leq n_{\beta}$ for every $\alpha<\beta$ in $\bar{A}$. Thus $V[G] \models \delta \in S_{0}(d)$. This contradicts Lemma 2.18. By Lemma 2.18 and the fact that $Q$ is $\kappa^{+\omega+1}$ - c.c., we get that $S$ is stationary in $V\left[G, G_{Q}\right]$, and therefore $\kappa^{+}-S_{0}(d)$ is stationary. By Fact 2.5 and Lemma 2.20 we get $V\left[G, G_{Q}\right] \models \neg A P_{\kappa}$ as required.

Proposition 2.21. $V\left[G, G_{Q}\right] \models V G S_{\kappa}$.

Proof. Let $\left\langle P_{n} \mid n<\omega\right\rangle$ be the supercompact Prikry sequence defined from $G_{Q}$, i.e., for each $m<\omega$, there is $p \in G_{Q}$ such that

$$
\left\langle P_{n} \mid n<m\right\rangle=\left\langle a_{0}^{p}, \ldots, a_{m-1}^{p}\right\rangle .
$$

Let $\kappa_{n}=P_{n} \cap \kappa$ for each $n<\omega$. Then $\left\langle\kappa_{n} \mid n<\omega\right\rangle$ is an increasing sequence of inaccessible cardinals cofinal in $\kappa$. Consider $\prod_{n<\omega} \kappa_{n}^{+\omega+1}$. For each $\alpha<\left(\kappa^{+\omega+1}\right)^{V}=\kappa^{+}$ and $n<\omega$ let $t_{\alpha}(n)=F_{\alpha}\left(\kappa_{n}\right)$ if $F_{\alpha}\left(\kappa_{n}\right)<\kappa_{n}^{+\omega+1}$ and $t_{\alpha}(n)=0$ otherwise. Clearly $\left\{t_{\alpha} \mid \alpha<\kappa^{+}\right\} \subseteq \prod_{n<\omega} \kappa_{n}^{+\omega+1}$. We show below that it is a scale and a very good one.

Claim 2.22. For each $\alpha<\beta<\kappa^{+}$we have $t_{\alpha}(n)<t_{\beta}(n)$ for all but finitely many $n$ 's.

Proof. Note that the set $Y=\left\{P \in P_{\kappa}\left(\kappa^{+\omega+2}\right) \mid F_{\alpha}(P \cap \kappa)<F_{\beta}(P \cap \kappa)<\right.$ $\left.(P \cap \kappa)^{+\omega+1}\right\} \in U^{*}$. Hence for each $n<\omega$ the projection $Y_{n}$ of $Y$ to $P_{\kappa}\left(\kappa^{+n}\right)$ belongs to $U_{n}$, i.e., the set $Y_{n}=\left\{P \cap \kappa^{+n} \mid P \in Y\right\} \in U_{n}$. By a simple density argument, we can find $q \in G_{Q}$ such that $X_{n}^{q} \subseteq Y_{n}$ for every $n \geq \ell(q)$. But by the choice of $Y_{n}, q$ forces that $t_{\alpha}(n)<t_{\beta}(n)<\kappa_{n}^{+\omega+1}$ for every $n \geq \ell(q)$ as required.

Claim 2.23. For each $t \in \prod_{n<\omega} \kappa_{n}^{+\omega+1}$ there exists $\alpha$ such that $t_{\alpha}(n)>t(n)$ for all but finitely many $n$ 's.

Proof. Let $\dot{t}$ be a name for $t$ and assume that $\Vdash \dot{t} \in \prod_{n<\omega} \kappa_{n}^{+\omega+1}$. Let us show that for every $q$ there is $q \leq^{*} p$ and $\alpha<\kappa^{+\omega+1}$ such that

$$
\Vdash t_{\alpha}(n)>t(n) \text { for almost every } n \text {. }
$$


Assume for simplicity that $\ell(q)=0$. Let $\vec{a}$ be as in Definition 2.11. Since $q \backslash\langle\vec{a}\rangle$ forces that $t(m)<(\vec{a}(m) \cap \kappa)^{+\omega+1}<\kappa$, we can use the Prikry condition and the fact that $\leq^{*}$ is $\kappa$ closed to find $r \geq^{*} q \succ\langle\vec{a}\rangle$, which determines the value of $\dot{t}(m)$. Using the same arguments as in the proof of the Prikry property, we can find $p^{\prime} \geq^{*} q$ such that for every $\vec{a}$ as in Definition 2.11 there exists $\beta_{\vec{a}}$ such that $p^{\prime}\langle\langle\vec{a}\rangle$ forces that $\dot{t}(m)=\beta_{\vec{a}}$. Let $h(\vec{a})=\beta_{\vec{a}}$. Note that for each $n$ we have

$$
j^{*}(h)\left(\kappa, j^{\prime \prime}\left(\kappa^{+}\right), \ldots, j^{\prime \prime}\left(\kappa^{+n}\right)\right)=\alpha_{n}<\kappa^{+\omega+1} .
$$

Let $\alpha=\sup \left\{\alpha_{n} \mid n<\omega\right\}+1$. By the construction of $F_{\alpha}$, we know that $j^{*}\left(F_{\alpha}\right)(\kappa)=$ $\alpha$, and so using (**), we can shrink the sets of measure one of $p^{\prime}$ to form a condition $p$ so that for every $\vec{a}, \beta_{\vec{a}}<F_{\alpha}(\vec{a}(m) \cap \kappa)$. It is simple to see that $\alpha$ and $p$ satisfy $(*)$.

Claim 2.24. $\left\langle t_{\alpha} \mid \alpha<\kappa^{+}\right\rangle$is a very good scale.

Proof. Let $\alpha<\kappa^{+}$be of uncountable cofinality below $\kappa$. Then $(c f \alpha)^{V\left[G, G_{Q}\right]}=$ $(c f \alpha)^{V[G]}=(c f(\alpha))^{V}$. Then pick a club $C \subseteq \alpha$ in $V$ with o.t. $(p(C))=c f \alpha$. Now by the choice of $U^{*}$ we have

$$
A=\left\{P \in \mathcal{P}_{\kappa}\left(\kappa^{+\omega+2}\right) \mid \forall \gamma, \beta \in C\left(\gamma<\beta \rightarrow F_{\gamma}(P \cap \kappa)<F_{\beta}(P \cap \kappa)\right\} \in U^{*}\right.
$$

since $j^{*}\left(F_{\gamma}\right)(\kappa)=\gamma<\beta=j^{*}\left(F_{\beta}\right)(\kappa)$ in $M\left[G^{*}\right]$ for each $\gamma<\beta<\left(\kappa^{+\omega+1}\right)^{V}$ and $|C|=c f \alpha<\kappa$.

Let $A_{n}$ be the projection of $A$ to $P_{\kappa}\left(\kappa^{+n}\right)$. The set of $q$ such that $X_{n}^{q} \subseteq A_{n}$ for every $n \geq \ell(q)$ is dense in $Q$ and so we can find such a condition $q$ in $G_{Q}$. Now it is simple to see that $q$ forces that $t_{\gamma}(m)<t_{\beta}(m)$ for every $m \geq \ell(q)$, and we are done.

Remark 2.25. (a) The same argument shows that $\left\langle t_{\alpha} \mid \alpha<\kappa^{++}=\left(\kappa^{+\omega+2}\right)^{V}\right\rangle$ is a very good scale in $\prod_{n<\omega} \kappa_{n}^{+\omega+2}$.

(b) It is possible instead of using the explicit construction producing the scale just to start with an indestructible under $\kappa$-directed closed forcing supercompact cardinal $\kappa$. Then set $2^{\kappa}=\kappa^{+\omega+2}$. Any functions $H_{\alpha}$ such that $\left[H_{\alpha}\right]_{\mathcal{V}}=\alpha\left(\alpha<\kappa^{+\omega+2}\right)$ with $\mathcal{V}$ being the projection of a supercompact measure from $\mathcal{P}_{\kappa}\left(\kappa^{+\omega+2}\right)$ to $\kappa$ can be used instead of the $F_{\alpha}$ 's.

(c) Cummings and Foreman have shown in an unpublished work that in $V^{Q}$ there is a scale on $\prod_{n} \kappa_{n}^{n+1}$ which is not good. This gives an alternative argument for the failure of $A P_{\kappa}$ in $V^{Q}$.

Our next task will be to push everything down to $\aleph_{\omega^{2}}$. The argument is quite standard, so let us only concentrate on the main points.

Let $j: V \rightarrow M$ be a $\kappa^{+\omega+1}$ supercompact embedding. We would like to find an extension $j^{*}$ of $j$ to $V[G]$ such that all the ordinals $\alpha<j(\kappa)$ will be of the form $j^{*}(g)(\kappa)$ for some $g: \kappa \rightarrow \kappa$.

Work in $V[G]$. Since ${ }^{\kappa^{+\omega+1}} M[G] \subseteq M[G]$ and the number of antichains of $j\left(P_{<\kappa}\right) / G$ in $M[G]$ is $\kappa^{+\omega+2}$, we can find a generic subset $H$ of $j\left(P_{<\kappa}\right) / G$ over $M[G]$. Set $M^{*}=M[G * H]$ and let $\left\langle x_{\alpha} \mid \alpha<\kappa^{+\omega+2}\right\rangle$ be an enumeration of $j(\kappa)$.

Lemma 2.26. There exists a generic subset $K$ of $C:=\left(C\left(j(\kappa), j\left(\kappa^{+\omega+1}\right)\right)\right)^{M[G * H]}$ with the following properties:

(a) $j^{\prime \prime}\left(G_{\kappa}\right) \subseteq K$;

(b) $j\left(F_{\alpha}\right)(\kappa)=x_{\alpha}$, where $F_{\alpha}$ is the $\alpha$-th Cohen function. 
Proof. Let $\left\langle A_{i} \mid i<\kappa^{+\omega+2}\right\rangle$ be an enumeration of the antichains of $C$ in $M^{*}$. Since $C$ is $\kappa^{+\omega+1}$ closed in $V[G]$, we can find a $C$ generic subset $K^{*}$ over $M^{*}$. For each $\alpha<j\left(\kappa^{+\omega+1}\right)$, set $K^{*} \uparrow_{\alpha}=\left\{\left.p\right|_{\alpha} \mid p \in K^{*}\right\}$. Set $F=\bigcup j^{\prime \prime}\left(G_{\kappa}\right)$. Note that $F \subseteq j^{\prime \prime}\left(\kappa^{+\omega+2}\right) \times \kappa \times \kappa$. For each $\alpha<j\left(\kappa^{+\omega+1}\right)$, we let $K \uparrow_{\alpha}$ be the set of all conditions $p$ such that for every $\delta<\kappa^{+\omega+2}$, if $j(\delta)<\alpha$, then $p(j(\delta)) \supseteq$ $j(F(\delta))=F(\delta)$ and $p(j(\delta))(\kappa)=x_{\delta}$. Note that since $\sup \left(j^{\prime \prime}\left(\kappa^{+\omega+2}\right)\right)=j\left(\kappa^{+\omega+2}\right)$, we need to change only $\kappa^{+\omega+1}$ many coordinates and so $p$ is in $M^{*}$. Since $K^{*} \uparrow_{\alpha}$ is $C \uparrow_{\alpha}:=(C(j(\kappa), \alpha))^{M^{*}}$ generic over $M^{*}$, and the number of changes is small (that is, $\left.\kappa^{+\omega+1}<j(\kappa)\right)$, we conclude that $K \uparrow_{\alpha}$ is also $(C(j(\kappa), \alpha))^{M^{*}}$ generic over $M^{*}$. Let $K=\bigcup_{\alpha<j\left(\kappa^{+\omega+2}\right)} K \uparrow_{\alpha}$. Since every antichain in $C$ is an antichain of $C \uparrow_{\alpha}$ for some $\alpha<j\left(\kappa^{+\omega+2}\right)$, we get that $K$ is $C$ generic over $M^{*}$. Also by our construction, $K$ satisfies (a) and (b) and we are done.

Let $j^{*}: V[G] \rightarrow M^{*}[K]$ be the extension of $j$ to $V[G]$. Let $U_{n}^{*}$ be the $\kappa^{+n}$ ultrafilter derived from $j^{*}$, i.e.,

$$
X \in U_{n}^{*} \text { iff } j^{\prime \prime}\left(\kappa^{+n}\right) \in j^{*}(X) .
$$

Let $i_{n}^{*}: V[G] \rightarrow U l t\left(V[G], U_{n}^{*}\right) \cong N_{n}$ and $k_{n}: N_{n} \rightarrow M^{*}[K]$. By standard arguments we can find an $M^{*}[K]$ generic subset $H^{*}$ of $\operatorname{Col}\left(\kappa^{+\omega+2}, j(\kappa)\right)$. Now by our construction, the range of $k_{n}$ contains $\left\{j^{*}\left(F_{\alpha}\right)(\kappa) \mid \alpha<\kappa^{+\omega+2}\right\} \cup\left\{k_{n}\left(i_{n}(\kappa)\right)\right\}=$ $j(\kappa)+1$ and so $\operatorname{crit}\left(k_{n}\right)>i_{n}(\kappa)$. But since $\left(\operatorname{Col}\left(\kappa^{+\omega+2}, i_{n}(\kappa)\right)\right)^{N_{n}}$ satisfies $i_{n}\left(\kappa^{+}\right)$c.c, the filter generated by $k_{n}^{-1}\left(H^{*}\right)$ is $\left(\operatorname{Col}\left(\kappa^{+\omega+2}, i_{n}(\kappa)\right)\right)^{N_{n}}$ generic over $N_{n}$. Denote this filter by $H_{n}$.

Now we are ready to define a new forcing $Q$.

Definition 2.27. $p \in Q$ iff

$$
p=\left\langle a_{0}^{p}, f_{0}^{p}, a_{1}^{p}, f_{1}^{p}, \ldots, a_{n-1}^{p}, f_{n-1}^{p} X_{n}^{p}, F_{n}^{p}, X_{n+1}^{p}, F_{n+1}^{p}, \ldots\right\rangle
$$

so that the following holds:

(1) $\left\langle a_{0}^{p}, a_{1}^{p}, \ldots, a_{n-1}^{p}, X_{n}^{p}, X_{n+1}^{p}, \ldots\right\rangle$ is as in Definition 2.9 with the $U_{n}^{*}$ 's replacing the $U_{n}$ 's.

(2) $\forall \ell<n-1 f_{\ell}^{p} \in \operatorname{Col}\left(\left(a_{\ell}^{p} \cap \kappa\right)^{+\omega+2}, a_{\ell+1}^{p} \cap \kappa\right)$.

(3) $f_{n-1}^{p} \in \operatorname{Col}\left(\left(a_{n-1}^{p} \cap \kappa\right)^{+\omega+2}, \kappa\right)$.

(4) $\forall \ell \geq n F_{n}$ is a function on $X_{n}^{p}$ such that

(a) $F_{n}(P) \in \operatorname{Col}\left((P \cap \kappa)^{+\omega+2}, \kappa\right)$.

(b) $j_{n}^{*}\left(F_{n}\right)\left(j_{n}^{\prime \prime} \kappa^{+}\right) \in H_{n}$.

All the previous claims remain valid here. Only in Lemma 2.16(b) do we restrict ourselves to $\mu$ 's of the form $\kappa_{n}^{+\omega+1}$ or $\kappa_{n}^{+\omega+2}$ for the Prikry sequence $\left\langle\kappa_{n} \mid n<\omega\right\rangle$.

Let us conclude with two questions.

Question 1. Is it consistent that $\aleph_{\omega}$ is a strong limit, $2^{\aleph_{\omega}}>\aleph_{\omega+1}$ and $\neg \square_{\aleph_{\omega}}^{*}$ (or $\left.\neg A P_{\aleph_{\omega}}\right)$ ?

Question 2. Is it is consistent that GCH holds below $\kappa, 2^{\kappa}>\kappa^{+}$and $\neg \square_{\kappa}^{*}$ (or $\neg A P_{\kappa}$ ) for a singular cardinal $\kappa$ ?

Question 3 (Cummings). Is it consistent that there is a very good scale on every increasing sequence $\left\langle\kappa_{n} \mid n<\omega\right\rangle$ of regular cardinals such that $\bigcup_{n<\omega} \kappa_{n}=\kappa$ and $\neg A P_{\kappa} ?$ 


\section{REFERENCES}

1. S. Ben-David and M. Magidor, The weak $\square^{*}$ is really weaker that the full square, J. Symbolic Logic 51 (1986) 1029-1033. MR.0865928 (88a:03117)

2. J. Cummings, M. Foreman and M. Magidor, Squares, scales and stationary reflection, Journal of Mathematical Logic 1 (2001), 35-98. MR.1838355 (2003a:03068)

3. J. Cummings and E. Schimmerling, Indexed squares, Israel Journal of Mathematics,131 (2002), 61-99. MR1942302 (2004k:03085)

4. M. Gitik and M. Magidor, Extender Based Forcing, J. of Symbolic Logic,59(2)(1994),445-460. MR:1276624 (95k:03079)

5. M. Gitik, Prikry Type Forcings, Handbook of Set Theory, eds. M. Foreman, A. Kanamori and M. Magidor,to appear. (www.math.tau.ac.il/ gitik)

6. T. Jech, Set Theory. The Third Millennium Edition, Springer 2003. MR.1940513 (2004g:03071)

7. A. Kanamori, The Higher Infinite, Springer 1994. MR.1321144 (96k:03125)

8. C. Merimovich, A Power Function with a Fixed Finite Gap Everywhere, J. of Symbolic Logic, to appear (arXiv:math.LO/0005179).

9. S. Shelah, On successors of singular cardinals, Logic Colloquium 78, eds. M. Boffa, D. van Dalen and K. MacAloon, (1979), 357-380. MR0567680 (82d:03079)

10. S. Shelah, Reflecting stationary sets and successors of singular cardinals, Archive Math. Logic 31 (1991), 25-53. MR.1126352 (93h:03072)

11. S. Shelah, Cardinal Arithmetic, Oxford University Press, Oxford (1994). MR.1318912 (96e:03001)

12. R. Solovay, Strongly compact cardinals and the GCH, Proceedings of the Tarski Symposium, Proceedings of Symposia in Pure Mathematics, vol.25, AMS (1974), 365-372. MR0379200 (52:106)

School of Mathematical Sciences, Raymond and Beverly Sackler Faculty of Exact Sciences, Tel Aviv University, Ramat Aviv 69978, Israel

E-mail address: gitik@post.tau.ac.il

School of Mathematical Sciences, Raymond and Beverly Sackler Faculty of Exact Sciences, Tel Aviv University, Ramat Aviv 69978, Israel

Current address: Department of Mathematics, University of California, Irvine, California 92717

E-mail address: sharona@math.uci.edu 